Introduction Musculoskeletal disorders (MSD) represent a significant occupational problem in intensive care unit (ICU) workers. This study aimed to determine ergonomic risk factors and the musculoskeletal symptoms in ICU workers in university hospital.

Methods Ergo team was created by the occupational health department in the hospital. The socio demographic data were obtained by a questionnaire. The clinical assessments were performed by a physiatrist. Cornell Musculoskeletal Discomfort Questionnaire was used for musculoskeletal symptoms assessment. Walk-through survey performed to determine main jobs and tasks in ICUs. The Rapid Entire Body Assessment (REBA) scale was used to assess the ergonomics risks for the nurses.

Results There were 30 patient $^{6}$ bed in both intensive care units. Twelve doctors(10.7\%), sixty four nurses (62.7\%) and twenty seven staff members $(26.2 \%)$ worked in two ICUs included in the study. Each nurse was responsible for two patients while the doctors and staff members were responsible entire units. 102 workers of those 56 from internal medicine ICU (IMICU) (56\%) and 46 from anaesthesia ICU (AICU) $(46 \%)$ workers were participated to the study. $60.7 \%$ of the study group was female and the mean age was $32 \pm 6.4$ in IMICU and $33.1+5.7$ in AICU $(p=0.3)$. According to the Cornell scale, $52(50.9 \%)$ had neck pain, 58 (56.8\%) had back pain and $25(24.5 \%)$ had wrist pain. Two ergonomically high risk tasks (patient positioning and working with monitor) were identified. The nurses' mean REBA score was $9.7+1.6$ in anaesthesia ICU and $8.7+2.0$ in internal medicine ICU $(p=0.8)$ for patient position taks. The mean REBA scores for anaesthesia ICU were $6.1+1.6$ and $4.8+1.4 \quad(\mathrm{p}=0.7)$ respectively.

Conclusion More than half of ICU workers had neck, back and wrist pain. ICU nurses had high ergonomics risks. In these units, 'ergonomic risk prevention programmes' should be implemented by occupational health teams.

\section{REDUCTION OF MUSCULOSKELETAL PAIN AMONG PROFESSIONAL MUSICIANS BY INTRODUCING RESISTANCE BAND TRAINING AT WORK} 1,2LPA Brandt*, ${ }^{1} \mathrm{MB}$ Panduro, ${ }^{2}$ SR Nielsen. ${ }^{1}$ Odense University Hospital, Odense Denmark;
${ }^{2}$ University of Southern Denmark, Odense, Denmark

\subsection{6/oemed-2018-ICOHabstracts.752}

Introduction Several studies have shown that professional symphony orchestra musicians have a higher prevalence of musculoskeletal complaints (MSC), compared to other work groups. MSC have a great impact on the musicians' workability and life quality, emphasising the importance of finding preventive measures

Methods A cross-sectional study, based on questionnaires before and after an intervention of resistance band training, among 350 musicians and administrative workers from 5 symphony orchestras in Denmark. The participant rated their degree of pain on a VAS scale from 0 to 10 and overall reduction of MSC. The changes from baseline to follow-up for each separate body region were evaluated using a linear mixed model.

Result MSC was most common in shoulders, neck, and lower back. Female musicians had a significantly higher prevalence of MSC than men. Low string group had the lowest prevalence of MSC, while high string, brass-winds and woodwind groups had a relative high prevalence of MSC. Resistance band training showed a positive significant effect with a mean reduction of VAS-scores in shoulders at -0.88 and neck at -0.52 . $45 \%$ of the participants experienced an overall reduction in musculoskeletal pain.

Discussion In compliance with the purpose, MSC before and after the intervention were assessed, showing a positive effect with regards to MSC in shoulders and neck. Future research should explore how realistic implementing resistance band training is as a steady regime among professional symphony orchestra musicians, likewise assuring a long lasting positive effect.

\section{STUDY ON WORK-RELATED MUSCULOSKELETAL SYMPTOMS AMONG TRAFFIC POLICE IN KATHMANDU VALLEY}

Leela Paudel. Kathmandu Medical College, Kathmandu University, Kathmandu, Nepal

\subsection{6/oemed-2018-ICOHabstracts.753}

Introduction Occupational environment plays an important role in health of the exposed population. Traffic police personnel (TPP) are more vulnerable to this situation. Workrelated Musculoskeletal symptoms (WRMSS) is defined as any trouble (ache, pain or discomfort) in nine topographic region of the body. The purpose of the study is to find the prevalence of WRMSS and to find the association between the various risk factors like-age, working hours, work in the field (years), body mass index(BMI), smoking, alcohol consumption, chewing tobacco with the development of WRMSS and to assess the sickness absenteeism and reduction in productivity.

Methods An analytical cross-sectional study of WRMMS was done among 355 traffic police from all 36 traffic booths of Kathmandu Valley. Data was collected using a questionnaires adapted from the Dutch and Nordic Musculoskeletal questionnaires. The number of traffic police was selected according to the proportion of the traffic police in each traffic booth.

Results The average age of the respondents with standard deviation were $29.59 \pm 6.99$ years and a majority were male (90.1\%). Almost 70\% of respondents had complain of WRMSS in any body parts during last 12 months and the most common site was low back (51.3\%). WRMSS lead to 9.25\% absenteeism from work and $2.25 \%$ change duties due to Musculoskeletal trouble. The variables age, duration of working hour, work in the field (years), BMI were significantly associated with WRMSS (all $\mathrm{p}<0.05)$. There was no significant association between education, smoking, alcohol consumption, and chewing tobacco with WRMSS in Traffic police.

Conclusion WRMSS affects more than 70\% of traffic police with most common site being the low back. Significant risk factors include- age, duration of working hour, work in the field (years) and body mass index. Periodic examination, ergonomics modification and health education will definitely help to improve the quality of life among this group.

\section{IDENTIFICATION OF INFLAMMATORY BIOMARKERS FOR THE EARLY DETECTION OF TENDONITIS DURING REPETITIVE MANUAL ASSEMBLY TASKS}

D Conde* ${ }^{*}$ G Ibarra-Mejia, JS Moore, K Browne. Universiy of Texas at El Paso, USA

10.1136/oemed-2018-ICOHabstracts.754 